Chapter 4

\title{
Acute Exacerbations of \\ Chronic Obstructive Pulmonary Disease
}

\author{
S. Uzun, R.S. Djamin, H.C. Hoogsteden, \\ J.G.J.V. Aerts and M.M. van der Eerden \\ Additional information is available at the end of the chapter \\ http://dx.doi.org/10.5772/54867
}

\section{Introduction}

Chronic obstructive pulmonary disease (COPD) is a disease which is characterized by airway inflammation and progressive airflow limitation with poor reversibility. Patients with COPD can experience periods of acute deterioration, which are called exacerbations. There are different definitions for an acute exacerbation of COPD (AECOPD). A symptom reported AECOPD is defined solely based on a patient's symptoms [1]. This is regardless of whether the patient seeks medical attention or receives treatment for the exacerbation. An event defined AECOPD requires a therapeutic intervention such as a change in COPD medications or a change in healthcare utilization [1]. Generally accepted is the definition as in the guidelines of the World Health Organization, US National Heart Lung and Blood Institute and Global Initiative for Chronic Obstructive Lung Disease (GOLD), which define an exacerbation as "an event in the natural course of the disease characterized by a change in the patient's baseline dyspnoea, cough, and/or sputum that is beyond normal day-to-day variations, is acute in onset and may warrant a change in regular medication in a patient with COPD" [2]. Frequent exacerbations can result in a decreased health related quality of life [3], a decline in lung function [4], an increased risk of hospitalization [5] and an increase in mortality [6].

COPD and acute exacerbations of COPD (AECOPD) impose a burden on health care and society. It is estimated that COPD is the $4^{\text {th }}$ leading cause of death worldwide and will be the $3^{\text {rd }}$ leading cause of death in 2030 [7]. Along with increasing mortality rates, the loss in disability-adjusted life years (DALYs) also rises. By 2030 COPD will be the $5^{\text {th }}$ leading cause of loss in DALYs globally, where it was only number 13 in 2004. Increasing health care costs will be the consequence of this trend. In the European Union COPD accounts for just over $3 \%$ of the total health care budget. In the USA, the direct and indirect costs for COPD are 
almost 50 billion USD. The majority of these costs are attributed to exacerbations [8]. The importance of exacerbations is reflected in the latest update of the GOLD report, in which the number of exacerbations in the preceding year is incorporated in the new classification of a patient with COPD [8]. In order to try to reduce the mortality, loss in DALYs and related costs and to lower the burden on society and health care, it is a goal to prevent and treat COPD and exacerbations of COPD. This chapter will give a concise overview of the background of AECOPD and the available tools for its treatment and prevention.

\section{Epidemiology}

The prevalence of COPD varies greatly per country and also within countries [9]. This heterogeneity can be contributed to not only differences in diagnostic methods and classification but also to smoking habits, population age, in- and outdoor air pollution, occupational exposure, prevalence of pulmonary tuberculosis, chronic asthma and socioeconomic status [10]. Prevalences of COPD have been reported varying from 0,2-37\% [11, 12]. The prevalence of AECOPD is very difficult to determine since there is no generally agreed definition for an AECOPD (see above). Studies show that only 32-50\% of symptom defined AECOPD are reported by patients to health care professionals [13, 14]. Although there is no reliable estimate of the prevalence of AECOPD, much is known about the occurrence of exacerbations. Research shows that exacerbations are more frequent in the winter season [15] and may occur clustered in time [16]. Exacerbations are also more frequent and severe as COPD severity increases [17]. Besides COPD severity, the history of exacerbations is also a good predictor of future exacerbations [17]. Furthermore, there is a strong correlation with symptoms of depression and recurrent exacerbations $[18,19]$.

\section{Pathophysiology of COPD and AECOPD}

COPD is the result of a chronic inflammation in the airways. The inflammation is initiated by chronic exposure to exogenic toxins (e.g. cigarette smoke) which is causing damage to the airway epithelium and is activating the innate immune system giving a rapid, nonspecific response [20, 21]. Of the innate immune response the neutrophillic inflammation is most prominent in COPD. The cells of the innate immune system activate the adaptive immune system, of which CD8+-cells, CD4+ $\mathrm{T}_{\text {helper }} 1$ cells and B-cells have an important role in COPD. This activation of the adaptive immune response is the beginning of a cascade which causes extensive chronic inflammation, oxidative stress and remodeling, resulting in destruction of alveolar space and deposition of connective tissue in the subepithelium and adventitium of the airway wall [22]. The degree of chronic inflammation in COPD correlates with the severity of airflow limitation. This is supported by a correlation which is seen between the severity of obstruction and presence of CD8+-cells and B-cells in the small conducting airways [22] and the presence of neutrophils in sputum [23]. Also, bacterial colonization is more frequently observed in patients with severe to very severe COPD, suggesting that bacterial colonization 
induces inflammation and contributes to the progression of COPD [24, 25]. The existence of the chronic inflammation and oxidative stress is supported by the presence of oxidants and numerous pro-inflammatory cytokines in the airways and serum. Compared to healthy controls, sputum specimens of patients with stable COPD and AECOPD show increased numbers of neutrophils and increased levels of pro-inflammatory cytokines like interleukin-6 (IL-6) and interleukin-8 (IL-8) [21, 23, 26-29]. During an AECOPD neutrophils, IL-6 and IL-8 are also increased in serum $[27,30,31]$. Interleukin-6 is a cytokine released during initial immune response by different cell types of the native immune system, like macrophages. It induces hepatic acute phase response during inflammation [32] which in turn increases production of C-reactive protein (CRP). Interleukin-6 is also a growth factor for T- and B-cells [33]. Interleuking- 8 is released by a variety of cell types involved in inflammation, like endothelial cells, fibroblasts and monocytes [34]. It is a potent neutrophil chemotactic and activating factor [34]. The presence of the increased inflammation in serum both during stable state and AECOPD may be explained by the "overspill theory", in which the local inflammatory processes in the lung "spill over" to the systemic circulation [35]. It is therefore thought that disease activity of COPD can be measured in serum by biomarkers. Exhaled breath condensate (EBC) components are thought to reflect the physiological state of lining fluid of the airways. It's a non-invasive mean of obtaining information on oxidative stress and inflammation in the airways. Hydrogen peroxide $\left(\mathrm{H}_{2} \mathrm{O}_{2}\right.$, a precursor of potent oxidants $\mathrm{OH}$ and $\mathrm{HOCl}$ ) and 8-isoprostane (formed by the free radical peroxidation of arachidonic acid) are EBC oxidative stress biomarkers proven to be elevated in patients with COPD during stable state and during exacerbations [31,36-38]. Heme-oxygenase-1 (HO-1) is an inducible catalyzer of the degradation of heme to biliverdin which is thought to provide protection from oxidative stress. It is decreased in ex-smokers with COPD compared to control subjects [39] but increased during severe exacerbations [29], in healthy smokers and current smokers with COPD [40].

\section{Aetiology of AECOPD}

\subsection{Microbiology}

There is a great variety in reported infectious causes of COPD exacerbations. It is of importance to determine, both for bacteria and viruses, whether the presence of the microbe is actually the cause of the exacerbation. Estimated is that about $50-78 \%$ of acute exacerbations of COPD are caused by respiratory infections [24, 27, 41, 42], in which the clinical presentation range from pneumonia to coryzal symptoms with dyspnea. Patients with AECOPD of proven infectious aetiology have a longer hospital stay and a greater decrease in $\mathrm{FEV}_{1}$ than patients with noninfective exacerbations [27].

\subsection{Viral causes}

In the past viruses have been an underestimated cause of AECOPD and the causative role of viruses in AECOPD is still not fully established. The observation that as well viral infections and exacerbations are seasonal does suggest that viruses have a role in AECOPD [15, 43]. 
Recently researchers deliberately exposed patients with COPD and healthy smokers to rhinoviruses and observed that this virus was able to cause an exacerbation in patients with COPD [44]. Detection of viruses by culture and by serology, where a second serum sample is also required, is less sensitive and more time consuming than PCR techniques, where only 1 sample is required. Because of the advanced PCR techniques in detecting viruses, the percentage of exacerbations they account for can also be overestimated. The presence of viral DNA or RNA does not implicate that the virus is the cause of an exacerbation as several studies reported patients with stable COPD to carry viruses with percentages varying from $12-19 \%$ $[45,46]$. In exacerbations several studies have reported that viruses were detected in $20-56 \%$ of cases [24, 27, 41, 42, 46, 47]. In these studies rhinovirus, influenza virus and respiratory syncytial virus and were the most common isolated viruses. A more extensive overview can be found in table 1 .

\begin{tabular}{|c|c|}
\hline \multicolumn{2}{|c|}{ COPD exacerbations: divided by cause } \\
\hline \multicolumn{2}{|c|}{ Bacteria } \\
\hline \multicolumn{2}{|r|}{ Streptococcus pneumoniae } \\
\hline \multicolumn{2}{|r|}{ Haemophilus influenzae } \\
\hline \multicolumn{2}{|r|}{ Moraxella catarrhalis } \\
\hline \multicolumn{2}{|r|}{ Haemophilus parainfluenzae } \\
\hline \multicolumn{2}{|r|}{ Pseudomonas aeruginosa } \\
\hline \multicolumn{2}{|r|}{ Staphylococcus aureus } \\
\hline \multicolumn{2}{|l|}{ Viruses } \\
\hline \multicolumn{2}{|r|}{ Human rhinovirus } \\
\hline \multicolumn{2}{|r|}{ Respiratory syncytial virus } \\
\hline \multicolumn{2}{|r|}{ Influenza virus } \\
\hline \multicolumn{2}{|r|}{ Parainfluenza virus } \\
\hline \multicolumn{2}{|r|}{ Human metapneuvirus } \\
\hline \multicolumn{2}{|r|}{ Coronavirus } \\
\hline \multicolumn{2}{|r|}{ Adenovirus } \\
\hline \multicolumn{2}{|c|}{ Atypical microorganisms } \\
\hline \multicolumn{2}{|r|}{ Mycoplasma pneumoniae } \\
\hline \multicolumn{2}{|r|}{ Chlamydophila pneumoniae } \\
\hline \multicolumn{2}{|r|}{ Legionella pneumophila } \\
\hline & Coxiella burnetii \\
\hline \multicolumn{2}{|l|}{ Other } \\
\hline \multicolumn{2}{|r|}{ Sulphur dioxide $\left(\mathrm{SO}_{2}\right)$} \\
\hline \multicolumn{2}{|r|}{ Ozone $\left(\mathrm{O}_{3}\right)$} \\
\hline \multicolumn{2}{|r|}{ Nitrogen dioxide $\left(\mathrm{NO}_{2}\right)$} \\
\hline & Particulate matter $\left(\mathrm{PM}_{2,5}, \mathrm{PM}_{10}\right)$ \\
\hline
\end{tabular}

Table 1. Most common causes of exacerbations of COPD. 


\subsection{Bacterial causes}

Bacteria as cause of AECOPD are reported from 30\% [48] up to 55\% [27, 49]. The most common bacterial pathogens are Streptococcus pneumoniae, Haemophilus influenzae, Moraxella catarrhalis and in patients with more severe COPD also Pseudomonas aeruginosa [42, 48]. It is difficult to determine the role of bacteria in AECOPD, as $34-48 \%$ of patients with COPD are reported to be colonized with bacteria [26, 27, 50,51]. Molecular typing of bacteria during exacerbations showed that the acquisition of new strains may cause exacerbations [52], but not every acquisition of a new strain is linked to an exacerbation.

\subsection{Non-microbial causes}

One tenth of AECOPD are due to environmental pollution, of which ozone, sulphur dioxide and nitrogen dioxide known and researched causes [53, 54]. Particulate matter (PM) is also related to increased admissions for COPD and other respiratory diseases [53, 55]. Particulate matter consists of a mixture of solid particles and liquid aerosols suspended in the air from natural sources, industrial activities and can also be traffic related [56]. Other possible, noninfectious causes may be left sided heart failure, change in environmental temperature, but about $30 \%$ of exacerbations are of unknown origin [6].

\section{Clinical Presentation and Diagnosis}

\subsection{History}

Patients with an AECOPD usually present with dyspnea, which may be acute but can also be a history of slowly progressive dyspnea. Coughing or sputum production may or may not be present. When expectorating sputum, it is important to assess whether sputum volume has increased and whether it is purulent (e.g. green). Purulent sputum is usually a sign of infection [57]. Fever or other signs of infection should be looked for. Hemoptysis may be present in case of a severe infection. Risk factors for atypical infections should be thought of.

\subsection{Laboratory tests}

Laboratory test can be performed if necessary. C-reactive protein as marker for inflammation can be performed. Additional laboratory tests can be performed depending on the differential diagnosis. If available, an arterial blood gas can be performed. Hypoxemia may be present and in more severe cases a patient can also retain $\mathrm{CO}_{2}$. Hypercapnia is defined as arterial blood gas $\mathrm{CO}_{2}\left(P_{\mathrm{a}} \mathrm{CO}_{2}\right)$ level above $45 \mathrm{mmHg}(6,00 \mathrm{kPa})$ and hypercapnic respiratory failure as $P_{\mathrm{a}} \mathrm{CO}_{2}$ of $>50 \mathrm{mmHg}(6,67 \mathrm{kPa})$. When present it is important to assess if the hypercapnia is longer existing and to assess if the patient is being able to metabolically compensate the hypercapnia. 


\subsection{Radiology}

A chest X-ray is mainly useful for excluding other pathology like pneumothorax, pleural fluid, congestive heart failure or otherwise. It may reveal consolidations or other pathology. In the acute phase a chest CT-scan has no additive value in the tract of diagnosing an exacerbation of COPD. It can be performed if doubts exist about the presence of pulmonary embolisms as an explanation for dyspnea and/or desaturation. In a patient with recurrent airways infections a CT-thorax can be performed to investigate whether bronchiectasis is present.

\subsection{Biomarkers}

Biomarkers can be used as indicators of a physiological state in which a patient is or may become, it can help in diagnosis, aetiology and prognosis. In theory, biomarkers could be used to predict exacerbations, to determine if a patient has increased inflammation, to distinguish type of inflammation (bacterial or viral infection or otherwise) or to predict clinical outcome after an AECOPD.

Many biomarkers have been researched of which many of them are of little clinical use. At this moment the most important biomarkers in AECOPD are CRP, serum IL-6, 8-isoprostane, $\mathrm{H}_{2} \mathrm{O}_{2}$ and procalcitonin (ProCT). These biomarkers are closely related to oxidative stress and inflammation. C-reactive protein is momentarily the most widely used marker of inflammation in clinical practice.

In patients with frequent exacerbations, both CRP and serum IL-6 levels are increased during a stable phase but also during the recovery period of an AECOPD [58,59] compared to patients with infrequent exacerbations. Interleukin- 6 is a cytokine which is widely expressed and produced in the body, and is not specific to the lung. Serum IL-6 has no additional value above CRP in clinical decision making. Interleukin-6 levels in sputum may be of use to predict therapy response [58], although more research is needed before clinical decisions can be made based on this biomarker. Similarly, there is a lack of studies which investigate the use of exhaled biomarkers 8-isoporstane and $\mathrm{H}_{2} \mathrm{O}_{2}$ for clinical purposes. Procalcitonin may be a biomarker which can discriminate in aetiology of an exacerbation but may also be used as therapeutic response parameter. Procalcitonin is the precursor of calcitonin and is released in response to a bacterial infection by many tissues under stimulation of several cytokines. Procalcitonin levels are minimally raised in viral infections [60], making it a relative specific diagnostic tool for bacterial infections. Most research has been performed in patients with community acquired pneumonia (CAP) [61]. It is suggested that ProCT could become a useful tool in clinical decision making regarding antibiotic therapy. There have been several trials to assess the utility of ProCT in AECOPD. In general ProCT-guided antibiotic therapy compared to standard management in AECOPD showed no differences in death from any cause, rates of intensive care unit (ICU) admission for any reason, duration of ICU stay, improvement of symptoms, difference in the quality-of-life score, re-exacerbation and readmission [62]. Procalcitonin-guided antibiotic therapy showed reduction in antibiotic prescription [62] and in one study [63] also reduction in antibiotic therapy duration, which in turn decreases the patient's exposure to antibiotics and related side effects, lowers the burden of antibiotic use and the risk of antimicrobial resistance. Procalcitonin is not yet being implemented in standard 
care though, as it is relatively expensive and there has been little to no research performed outside Europe.

\section{Management}

The treatment of an AECOPD consists of supportive therapy, maximal bronchodilation, steroids to reduce the inflammation and treatment of the cause.

\subsection{Supportive therapy}

Oxygen delivery is one of the first supportive therapies which can be provided for a patient. Oxygen saturation should be at least $90 \%$ though in some cases lower saturations may also be accepted. Too much oxygen may cause hypercapnia as the drive to breathe in some COPD patients may rely on arterial $\mathrm{O}_{2}$ pressure. Symptoms of acute hypercapnia are somnolence, headache, drowsiness, confusion, flushed skin or agitation. Physiotherapy during an admission for an AECOPD can prevent deterioration in skeletal muscle function and improve exercise capacity $[64,65]$. Because an AECOPD is accompanied by an impaired energy balance due to a decreased dietary intake and an increased resting energy expenditure, nutritional support may also benefit the patient in terms of general well-being and prevention of muscle wasting [66-68].

\subsection{Pharmacotherapy}

An exacerbation is the result of increased inflammation causing increased flow limitation. Treatment should be directed towards controlling this exacerbated inflammation and maximizing bronchodilation. Short acting agents like salbutamol and ipratropium are mostly used for maximal bronchodilation, usually delivered by nebulizer. Many patients may not be able to generate the flows required to use other devices during an exacerbation. Corticosteroids have been proven to reduce time to recovery and treatment failure, increase $\mathrm{FEV}_{1}$ and arterial hypoxemia [8]. Treatment schemes have been reported varying from $30 \mathrm{mg}$ prednisolone orally to $60 \mathrm{mg}$ intravenous, ranging from 5 days to two weeks. Studies showed that there is no difference in clinical outcome if a patient is treated with oral steroids compared to parenteral steroids [69]. Antibiotic treatment can be initiated when a bacterial infection is suspected. With the Anthonisen criteria [70] one can decide whether antibiotic treatment is necessary or not. These criteria are derived from a randomized placebo-controlled crossover trial which has been performed in the ' 80 s where patients with COPD exacerbations were treated with antibiotics or placebo. The cardinal symptoms of infection in this study were increased sputum volume and purulence in combination with increased dyspnea. An exacerbation with all the previous 3 symptoms is called a type 1 exacerbation; two out of three symptoms have to be present for a type 2 exacerbation; one out of three and at least one other "minor symptom" (see table 2) have to be present for it to be a type 3 exacerbation. Patients with type 1 and type 2 exacerbations are most likely to benefit from antibiotic therapy. In Spain a pilot study was performed with hospitalized patients with AECOPD, where antibiotic therapy was given to 
patients with self-reported purulent sputum and withheld in patients with non-purulent sputum [71]. There was no difference between the two groups in treatment failure on day 3 , suggesting patient reported non-purulent sputum may be a valid criterion to withhold antibiotics [71]. A Dutch study showed that addition of doxycycline to the treatment regimen with glucocorticoids of a patient with an exacerbation was superior on day 10 but equivalent on day 30 in terms of clinical success and clinical cure compared to glucocorticoids alone, even in patients not showing signs of infection [72]. Most recently Spanish investigators performed a multicenter trial where they suggested that treatment of a mild to moderate exacerbation with amoxicillin/clavulanate, independent of glucocorticoids treatment, might give better clinical cure after 10 days compared to placebo [73]. In this study the median time to next exacerbation was also increased in patients receiving antibiotics compared to placebo. Unfortunately, because of recruitment problems this study did not reach the calculated amount of patients needed, so that definite conclusions cannot be made from the results of this study.

\begin{tabular}{ll}
\hline $\begin{array}{l}\text { Classification of AECOPD according to } \\
\text { Anthonisen criteria }\end{array}$ & Presence of symptoms and findings \\
\hline Type 1 & $\begin{array}{l}\text { Increased dyspnea } \\
\text { Increased sputum volume } \\
\text { Increased sputum purulence }\end{array}$ \\
\hline Type 2 & Two symptoms of type 1 \\
\hline Type 3 & 1 of three symptoms of type 1, plus at least one of the following \\
& findings: \\
& - Upper respiratory infection (sore throat, nasal discharge) within the \\
& past 5 days \\
& - Fever without other cause \\
& - Increased wheezing \\
& - Increased cough \\
& - Increase in respiratory rate or heart rate by 20\% as compared with \\
& baseline \\
\hline
\end{tabular}

Table 2. Classification of acute exacerbations of COPD according to Anthonisen criteria [70]

\section{Prevention}

Preventing exacerbations is an important treatment goal in COPD. There is a wide range of preventive measures which have proven to reduce exacerbation frequency or hospitalization in patients with AECOPD.

\subsection{Supportive measures}

Influenza vaccination and pneumococcal vaccination have both been researched as preventive measures for infection associated exacerbations. Current GOLD guidelines [8] advise influenza vaccination for patients with COPD. Pneumococcal vaccination is mainly advised for elderly 
patients with COPD. Investigation on this subject is ongoing. Of the non-pharmacologic interventions, pulmonary rehabilitation is the most effective in reducing hospital admissions and mortality and improving health-related quality of life in COPD patients who have recently suffered an exacerbation of COPD [74].

\subsection{Long-acting bronchodilators}

Long-acting bronchodilators can be divided in two groups: long acting muscarinic receptor antagonists (LAMAs) and long acting $\beta$-agonists (LABAs). Both have proven to show a positive effect on exacerbation reduction and improvement in quality of life [75-79]. An overview of the long-acting bronchodilators is given in table 3.

\begin{tabular}{ll}
\hline LABA & LAMA \\
\hline Formoterol & Tiotropium \\
\hline Arfomoterol & Glycopyrronium \\
\hline Salmeterol & \\
\hline Indacaterol & \\
\hline
\end{tabular}

Table 3. An overview of available long-acting bronchodilators.

Of the long-acting bronchodilators, indacaterol and glycopyrronium are the most recent additions for the treatment of COPD. Indacaterol is proven to be superior to formoterol, salmeterol and tiotropium in terms of use of rescue medication, dyspnoea score and health related quality of life. Compared to salmeterol and formoterol it is also superior in improving spirometry values. Indacaterol is non-inferior to tiotropium but when added to tiotropium therapy it is superior compared to tiotropium alone [80]. Indacaterol also lowers the risk of AECOPD compared to placebo [78, 81, 82]. Glycopyrronium has been approved in 2012 as therapy for COPD. It provides significant improvements in lung function, dyspnoea, health status, exacerbation frequency and rescue medication use versus placebo, and is comparable to tiotropium [83, 84]. The combination of glycopyrronium and indacaterol has shown superiority in bronchodilation compared to indacaterol alone [85]. Glycopyrronium has not been compared to other LABAs yet.

\subsection{Inhalation corticosteroids}

Inhalation corticosteroids (ICS) can be given to patients with high risk of exacerbations. In several studies ICS provided a reduction of symptoms (dyspnea, cough) and reduced the frequency of exacerbations [86-88]. The GOLD guidelines advise treatment for high exacerbation risk patients with few symptoms (group C) with a combination of ICS/LABA or a LAMA alone, or a combination of LABA and LAMA [8]. For high exacerbation risk patients who have many symptoms (group D) the same treatment is advised as for group C, also a combination of all three classes of inhalation drugs is possible [8]. 


\subsection{Phosphodiesterase inhibitors}

Currently, two phosphodiesterase inhibitors are available for the treatment of COPD: theophylline and roflumilast. Theophylline is a xanthine derivative which acts as a non-selective phosphodiesterase inhibitor. It has bronchodilator effects, improves symptoms and there is evidence that it can reduce exacerbations [89-91]. It is a drug which needs therapeutic window monitoring. It can interact with many drugs and can have toxic side effects which may be potentially dangerous, like cardiac arrhythmia. Therapy with theophyllines is not recommended if LABAs are available but can be used as add-on therapy [8]. Roflumilast is a selective phosphodiesterase-4 inhibitor. It increases prebronchodilator FEV1 and can reduce exacerbations in a selected group of patients with COPD $[92,93]$. In all trials patients in the roflumilast group experienced more side effects in comparison to patients in the placebo groups. The side effects were mostly gastro-intestinal related (nausea, diarrhoea, weight loss) and headache. These adverse events were associated with increased patient withdrawal in the roflumilast groups. The design of the trials limits the generalizability of these results. The included COPD patients were required to have symptoms of chronic bronchitis and AECOPD in the past. More investigation is needed to determine the exact place of this medication in the treatment of AECOPD.

\subsection{Macrolide antibiotics}

Antibiotic prevention of exacerbations is a highly researched topic in COPD. The most promising class of antibiotics appear to be macrolides. In various chronic lung diseases they seem to have an immune modulatory function.

\subsubsection{Proposed working mechanism}

Much in vivo and in vitro research has been performed with macrolide antibiotics. The effects of macrolides can be divided in antimicrobial effects and immune modulatory effects. Macrolides bind to the 505 subunit of the bacterial ribosome and inhibit bacterial protein synthesis [94]. Most macrolides have a uniform degree of activity; their antimicrobial spectrum extends from Gram-positive bacteria to a limited activity against Gram-negative bacteria [95]. Of the macrolides, azithromycin displays superior activity against Gram-negative organisms, such as H. influenzae [94]. Compared to other macrolides as erythromycin and clarithromycin, azithromycin also has better uptake in peripheral blood polymorphonuclear neutrophils (PMN) with slower release [96, 97], better tissue uptake and tissue concentrations are higher long after the last administered dose [98, 99]. Pseudomonas aeruginosa is a Gram-negative rod which has intrinsic resistance for macrolides but has nonetheless been extensively studied in combination with macrolides. Studies have shown that macrolides influence the virulence of not only P. aeruginosa [100-102] but also other microorganisms, like Proteus mirabilis [103], Salmonella enterica [104], Staphylococcus epidermidis [105] and H. influenzae [106]. Macrolides alter the biofilm around bacteria [105-107], in P. aeruginosa this may facilitate phagocytosis by PMN [101]. It is also suggested that macrolides block quorum sensing [108, 109] in P. aeruginosa, reduce flagellin synthesis and expression [103, 104] and reduce production of bacterial exoenzymes [100]. In murine models and in in vitro studies macrolides have shown to 
influence respiratory viral infections. In one study therapy with erythromycin increased survival rates in mice infected with lethal doses of influenza virus [110]. This effect might be exerted through the inhibitory action of erythromycin against virus-induced inflammatory responses in the lung. The production of interferon-gamma (IFN- $\gamma$ ) in the lungs was significantly decreased by the administration of erythromycin to the infected mice. Two in vitro studies researching the effect of erythromycin and clarithromycin in human tracheal cells infected with rhinovirus and influenza A virus, also showed that macrolides decrease the production of pro-inflammatory cytokines and inhibited activation of nuclear factor- $\mathrm{kB}$ ( $\mathrm{a}$ regulating factor in transcription of DNA in response to cellular stress) [111, 112]. These antiviral effects of macrolides have not yet been proven in patients, although there is evidence that macrolides may prevent common colds which are mostly of viral aetiology [113]. Macrolides support the airway innate immune system by maintaining airway epithelial integrity $[114,115]$. In vitro [116] and in vivo [117] studies show that macrolides improve alveolar macrophage phagocytosis function. Macrolide therapy stimulates the prolonged degranulation of neutrophils (suggesting anti-inflammatory activity in non-infective inflammation), decreases long term oxidative burst and can decrease the release of pro-inflammatory cytokines (such as IL-6 and IL-8) in healthy individuals [118]. In vitro it is observed that macrolides decrease the release of IL-1 $\beta$, IL- 6 and tumor necrosis factor-alpha (TNF- $\alpha$ ) in sputum cells of patients with COPD [119]. Azithromycin exerts direct inhibitory effects on mucus secretion from airway epithelial cells in vitro and in vivo [120].

\subsubsection{Success of macrolides in chronic lung diseases}

Diffuse panbronchiolitis is a progressive inflammatory disorder of the airways found almost exclusively in Japan. Clinically it is characterized by chronic cough, excessive sputum production, exertional breathlessness, chronic sinusitis and Pseudomonas colonization [121]. Untreated, the prognosis of diffuse panbronchiolitis is poor, with progressive deterioration of lung function, the development of diffuse bronchiectasis and death caused by respiratory failure. The introduction of long-term macrolide therapy has resulted in dramatic improvements in survival, with 5-year survival rates increasing from 63 to $92 \%$ [121, 122]. Significant symptom reduction and improved pulmonary function have also been achieved [123-126]. Also in patients with cystic fibrosis (CF) who are colonized with P. aeruginosa, macrolide therapy had led to improvement in $\mathrm{FEV}_{1}$ and forced vital capacity (FVC), a reduction in exacerbation rate, a reduction in hospital days and days of intravenous antibiotic use, delaying time until the first exacerbation and reducing number of additional courses of antibiotics [127-132]. A Cochrane review of macrolide therapy concluded that treatment with azithromycin had a small but significant effect on pulmonary function in patients with cystic fibrosis [133]. In a in New Zealand performed randomized controlled trial in non-CF bronchiectasis, maintenance treatment with 3 times a week $500 \mathrm{mg}$ azithromycin showed a reduction in exacerbations [134] though no effects were seen in quality of life and lung function. In a Dutch study where a treatment scheme was given with daily $250 \mathrm{mg}$ azithromycin, the reduction in exacerbations was accompanied by an improved quality of life assessed by St George's Respiratory Questionnaire (SGRQ) and an increase in lung function [135]. As for COPD there have been few researches concerning macrolides in preventing AECOPD. One published study 
has examined the effect of clarithromycin treatment in COPD [136]. This was a prospective double-blind randomized controlled trial of 67 patients with moderately severe COPD. The effects of 3 months' clarithromycin therapy on health status, exacerbation rate and sputum bacterial numbers were measured. Overall, no significant benefit was seen in any measure. However, significant improvements in both the SGRQ symptom score and 36-item short-form health survey (SF-36) physical function score were seen. A Japanese study performed in 1997 investigated the effect of long-term erythromycin therapy on common colds in patients with COPD [113]. It was a prospective, randomized, controlled but not blinded study. Patients who received erythromycin therapy had less common colds and less subsequent AECOPD compared to patients in the control group. In 2006, another study had been performed in the UK to investigate the influence of erythromycin on exacerbation of COPD [137]. Unfortunately, the total number of patients needed for inclusion was not reached. Although the study showed a significant reduction in number of exacerbations in COPD patients who received 1 year daily erythromycin, the reached conclusions should be carefully interpreted. The most recent study published concerning long term macrolide therapy in COPD was performed in the USA with over 1,000 patients. It showed a reduction in time to first exacerbation and a reduced risk for exacerbations in patients receiving daily azithromycin during 1 year [138]. The study participants were patients who had at least 1 treated exacerbation in the previous year or who were on continuous supplemental oxygen or had an emergency department (ED) visit or hospital admission for an exacerbation COPD. The applicability of these results is somewhat difficult. The results of the study could suggest that long term azithromycin can be given to many COPD patients, even to those who are not actually frequent exacerbators. The place of azithromycin in the prevention of COPD exacerbations is a topic which needs further research.

\subsubsection{Antimicrobial resistance}

Giving long term antibiotic treatment to a patient may have consequences; the development of antimicrobial resistance is by far the most important one. Several researches have shown that the erm(B) and mef genes are mostly responsible for macrolide resistance in streptococci bacteria [139-142]. This resistance can develop even when short term therapy with macrolides is given [141]. The participants receiving azithromycin in the USA study, where a 1-year therapy was administered, were less likely to be colonized with respiratory pathogens but more likely to become colonized with macrolide resistant pathogens [138]. In the UK study in patients with COPD the researchers found there were no significant changes in resistance of sputum pathogens (H. influenzae, S. pneumoniae, M. catarrhalis) after 1 year of daily erythromycin [137]. In a Dutch study investigating antibiotic treatment before cardiovascular surgery 300 patients were treated with 2 weeks of clarithromycin. A significant rise in macrolide resistance in oropharyngeal flora was observed and this resistance continued to exist for at least 8 weeks [140]. Since macrolide resistance in pneumococci is already a known problem $[141,143,144]$ it is of great importance to prevent the development of resistance in other microorganisms. 


\section{Author details}

S. Uzun ${ }^{1}$, R.S. Djamin ${ }^{1}$, H.C. Hoogsteden ${ }^{2}$, J.G.J.V. Aerts ${ }^{1,2}$ and M.M. van der Eerden ${ }^{2}$

1 Department of Respiratory Medicine, Amphia Ziekenhuis, Breda, The Netherlands

2 Department of Respiratory Medicine, Erasmus Medical Centre, Rotterdam, The Netherlands

\section{References}

[1] Pauwels, R, et al. COPD exacerbations: the importance of a standard definition. Respir Med, (2004). , 99-107.

[2] Rabe, K. F, et al. Global strategy for the diagnosis, management, and prevention of chronic obstructive pulmonary disease: GOLD executive summary. Am J Respir Crit Care Med, (2007). , 532-555.

[3] Seemungal, T. A, et al. Effect of exacerbation on quality of life in patients with chronic obstructive pulmonary disease. Am J Respir Crit Care Med, (1998). Pt 1): , 1418-1422.

[4] Donaldson, G. C, et al. Relationship between exacerbation frequency and lung function decline in chronic obstructive pulmonary disease. Thorax, (2002). , 847-852.

[5] Terzano, C, et al. Comorbidity, hospitalization, and mortality in COPD: results from a longitudinal study. Lung, (2010). , 321-329.

[6] Connors, A. F, et al. Outcomes following acute exacerbation of severe chronic obstructive lung disease. The SUPPORT investigators (Study to Understand Prognoses and Preferences for Outcomes and Risks of Treatments). Am J Respir Crit Care Med, (1996). Pt 1): , 959-967.

[7] Mathers, C, \& Ma, B. T. Fat D, Global Burden of Disease: 2004 update, (2008). World Health Organization. , 146.

[8] Vestbo, J. Global strategy for the diagnosis, management, and prevention of chronic obstructive pulmonary disease (revised 2011), (2011). Global Initiative for Chronic Obstructive Lung Disease. , 78.

[9] Buist, A. S, Vollmer, W. M, \& Mcburnie, M. A. Worldwide burden of COPD in highand low-income countries. Part I. The burden of obstructive lung disease (BOLD) initiative. Int J Tuberc Lung Dis, (2008). , 703-708.

[10] Salvi, S. S, \& Barnes, P. J. Chronic obstructive pulmonary disease in non-smokers. Lancet, (2009). , 733-743. 
[11] Atsou, K, Chouaid, C, \& Hejblum, G. Variability of the chronic obstructive pulmonary disease key epidemiological data in Europe: systematic review. BMC Med, (2011). , 7 .

[12] Rycroft, C. E, et al. Epidemiology of chronic obstructive pulmonary disease: a literature review. Int J Chron Obstruct Pulmon Dis, (2012). , 457-494.

[13] Langsetmo, L, et al. Underreporting exacerbation of chronic obstructive pulmonary disease in a longitudinal cohort. Am J Respir Crit Care Med, (2008). , 396-401.

[14] Seemungal, T. A, et al. Time course and recovery of exacerbations in patients with chronic obstructive pulmonary disease. Am J Respir Crit Care Med, (2000). , 1608-1613.

[15] Jenkins, C. R, et al. Seasonality and determinants of moderate and severe COPD exacerbations in the TORCH study. Eur Respir J, (2012). , 38-45.

[16] Hurst, J. R, et al. Temporal clustering of exacerbations in chronic obstructive pulmonary disease. Am J Respir Crit Care Med, (2009). , 369-374.

[17] Hurst, J. R, et al. Susceptibility to exacerbation in chronic obstructive pulmonary disease. N Engl J Med, (2010). , 1128-1138.

[18] Ito, K, et al. Depression, but not sleep disorder, is an independent factor affecting exacerbations and hospitalization in patients with chronic obstructive pulmonary disease. Respirology, (2012). , 940-949.

[19] Papaioannou, A. I, et al. The impact of depressive symptoms on recovery and outcome of hospitalised COPD exacerbations. Eur Respir J, (2012).

[20] Cosio, M. G, Saetta, M, \& Agusti, A. Immunologic aspects of chronic obstructive pulmonary disease. N Engl J Med, (2009). , 2445-2454.

[21] Baines, K. J, Simpson, J. L, \& Gibson, P. G. Innate immune responses are increased in chronic obstructive pulmonary disease. PLoS One, (2011). , e18426.

[22] Hogg, J. C, et al. The nature of small-airway obstruction in chronic obstructive pulmonary disease. N Engl J Med, (2004). , 2645-2653.

[23] Keatings, V. M, et al. Differences in interleukin-8 and tumor necrosis factor-alpha in induced sputum from patients with chronic obstructive pulmonary disease or asthma. Am J Respir Crit Care Med, (1996). , 530-534.

[24] Sethi, S, \& Murphy, T. F. Infection in the pathogenesis and course of chronic obstructive pulmonary disease. N Engl J Med, (2008). , 2355-2365.

[25] Marin, A, et al. Effect of Bronchial Colonisation on Airway and Systemic Inflammation in Stable COPD. COPD, (2012).

[26] Wilkinson, T. M, et al. Effect of interactions between lower airway bacterial and rhinoviral infection in exacerbations of COPD. Chest, (2006). , 317-324. 
[27] Papi, A, et al. Infections and airway inflammation in chronic obstructive pulmonary disease severe exacerbations. Am J Respir Crit Care Med, (2006). , 1114-1121.

[28] Bhowmik, A, et al. Relation of sputum inflammatory markers to symptoms and lung function changes in COPD exacerbations. Thorax, (2000). , 114-120.

[29] Tsoumakidou, $M$, et al. Nitrosative stress, heme oxygenase-1 expression and airway inflammation during severe exacerbations of COPD. Chest, (2005). , 1911-1918.

[30] Hurst, J. R, et al. Systemic and upper and lower airway inflammation at exacerbation of chronic obstructive pulmonary disease. Am J Respir Crit Care Med, (2006). , 71-78.

[31] Gerritsen, W. B, et al. Markers of inflammation and oxidative stress in exacerbated chronic obstructive pulmonary disease patients. Respir Med, (2005). , 84-90.

[32] Yamamoto, K, \& Rose-john, S. Therapeutic blockade of interleukin-6 in chronic inflammatory disease. Clin Pharmacol Ther, (2012). , 574-576.

[33] Chung, K. F. Cytokines in chronic obstructive pulmonary disease. Eur Respir J Suppl, (2001). , 50s-59s.

[34] Smith, W. B, et al. Interleukin-8 induces neutrophil transendothelial migration. Immunology, (1991). , 65-72.

[35] Sinden, N. J, \& Stockley, R. A. Systemic inflammation and comorbidity in COPD: a result of'overspill' of inflammatory mediators from the lungs? Review of the evidence. Thorax, (2010). , 930-936.

[36] Dekhuijzen, P. N, et al. Increased exhalation of hydrogen peroxide in patients with stable and unstable chronic obstructive pulmonary disease. Am J Respir Crit Care Med, (1996). Pt 1): , 813-816.

[37] Antczak, A, et al. Exhaled eicosanoids and biomarkers of oxidative stress in exacerbation of chronic obstructive pulmonary disease. Arch Med Sci, (2012). , 277-285.

[38] Biernacki, W. A, Kharitonov, S. A, \& Barnes, P. J. Increased leukotriene B4 and 8-isoprostane in exhaled breath condensate of patients with exacerbations of COPD. Thorax, (2003). , 294-298.

[39] Maestrelli, $P$, et al. Decreased haem oxygenase-1 and increased inducible nitric oxide synthase in the lung of severe COPD patients. Eur Respir J, (2003). , 971-976.

[40] Maestrelli, P, et al. Increased expression of heme oxygenase (HO)-1 in alveolar spaces and HO-2 in alveolar walls of smokers. Am J Respir Crit Care Med, (2001). Pt 1): , 1508-1513.

[41] Sapey, E, \& Stockley, R. A. COPD exacerbations. 2: aetiology. Thorax, (2006). , 250-258.

[42] Wedzicha, J. A, \& Seemungal, T. A. COPD exacerbations: defining their cause and prevention. Lancet, (2007). , 786-796. 
[43] Fisman, D. Seasonality of viral infections: mechanisms and unknowns. Clin Microbiol Infect, (2012). , 946-954.

[44] Mallia, P, et al. Experimental rhinovirus infection as a human model of chronic obstructive pulmonary disease exacerbation. Am J Respir Crit Care Med, (2011). , 734-742.

[45] Seemungal, T, et al. Respiratory viruses, symptoms, and inflammatory markers in acute exacerbations and stable chronic obstructive pulmonary disease. Am J Respir Crit Care Med, (2001). , 1618-1623.

[46] Rohde, G, et al. Respiratory viruses in exacerbations of chronic obstructive pulmonary disease requiring hospitalisation: a case-control study. Thorax, (2003). , 37-42.

[47] Ko, F. W, et al. Viral etiology of acute exacerbations of COPD in Hong Kong. Chest, (2007). , 900-908.

[48] Ko, F. W, et al. A 1-year prospective study of the infectious etiology in patients hospitalized with acute exacerbations of COPD. Chest, (2007). , 44-52.

[49] Bafadhel, M, et al. Acute exacerbations of chronic obstructive pulmonary disease: identification of biologic clusters and their biomarkers. Am J Respir Crit Care Med, (2011). , 662-671.

[50] Sethi, S, et al. Airway inflammation and bronchial bacterial colonization in chronic obstructive pulmonary disease. Am J Respir Crit Care Med, (2006). , 991-998.

[51] Zhang, M, et al. Relevance of lower airway bacterial colonization, airway inflammation, and pulmonary function in the stable stage of chronic obstructive pulmonary disease. Eur J Clin Microbiol Infect Dis, (2010). , 1487-1493.

[52] Sethi, S, et al. New strains of bacteria and exacerbations of chronic obstructive pulmonary disease. N Engl J Med, (2002). , 465-471.

[53] Ko, F. W, et al. Temporal relationship between air pollutants and hospital admissions for chronic obstructive pulmonary disease in Hong Kong. Thorax, (2007). , 780-705.

[54] Sint, T, Donohue, J. F, \& Ghio, A. J. Ambient air pollution particles and the acute exacerbation of chronic obstructive pulmonary disease. Inhal Toxicol, (2008). , 25-29.

[55] Qiu, H, et al. Effects of coarse particulate matter on emergency hospital admissions for respiratory diseases: a time-series analysis in Hong Kong. Environ Health Perspect, (2012). , 572-576.

[56] Valavanidis, A, Fiotakis, K, \& Vlachogianni, T. Airborne particulate matter and human health: toxicological assessment and importance of size and composition of particles for oxidative damage and carcinogenic mechanisms. J Environ Sci Health C Environ Carcinog Ecotoxicol Rev, (2008). , 339-362. 
[57] Stockley, R. A, et al. Relationship of sputum color to nature and outpatient management of acute exacerbations of COPD. Chest, (2000). , 1638-1645.

[58] Perera, W. R, et al. Inflammatory changes, recovery and recurrence at COPD exacerbation. Eur Respir J, (2007). , 527-534.

[59] Agusti, A, et al. Persistent systemic inflammation is associated with poor clinical outcomes in COPD: a novel phenotype. PLoS One, (2012). , e37483.

[60] Linscheid, P, et al. In vitro and in vivo calcitonin I gene expression in parenchymal cells: a novel product of human adipose tissue. Endocrinology, (2003). , 5578-5584.

[61] Schuetz, P, Amin, D. N, \& Greenwald, J. L. Role of procalcitonin in managing adult patients with respiratory tract infections. Chest, (2012). , 1063-1073.

[62] Tokman, S, Schuetz, P, \& Bent, S. Procalcitonin-guided antibiotic therapy for chronic obstructive pulmonary disease exacerbations. Expert Rev Anti Infect Ther, (2011). , 727-735.

[63] Schuetz, P, et al. Effect of procalcitonin-based guidelines vs standard guidelines on antibiotic use in lower respiratory tract infections: the ProHOSP randomized controlled trial. JAMA, (2009). , 1059-1066.

[64] Troosters, T, et al. Resistance training prevents deterioration in quadriceps muscle function during acute exacerbations of chronic obstructive pulmonary disease. Am J Respir Crit Care Med, (2010). , 1072-1077.

[65] Kirsten, D. K, et al. Exercise training improves recovery in patients with COPD after an acute exacerbation. Respir Med, (1998). , 1191-1118.

[66] Vermeeren, M. A, Schols, A. M, \& Wouters, E. F. Effects of an acute exacerbation on nutritional and metabolic profile of patients with COPD. Eur Respir J, (1997). , 2264-2269.

[67] Vermeeren, M. A, et al. Nutritional support in patients with chronic obstructive pulmonary disease during hospitalization for an acute exacerbation; a randomized controlled feasibility trial. Clin Nutr, (2004). , 1184-1192.

[68] Saudny-unterberger, H, Martin, J. G, \& Gray-donald, K. Impact of nutritional support on functional status during an acute exacerbation of chronic obstructive pulmonary disease. Am J Respir Crit Care Med, (1997). Pt 1): , 794-799.

[69] De Jong, Y. P, et al. Oral or IV prednisolone in the treatment of COPD exacerbations: a randomized, controlled, double-blind study. Chest, (2007). , 1741-1747.

[70] Anthonisen, N. R, et al. Antibiotic therapy in exacerbations of chronic obstructive pulmonary disease. Ann Intern Med, (1987). , 196-204.

[71] Soler, N, et al. Sputum purulence-guided antibiotic use in hospitalised patients with exacerbations of COPD. Eur Respir J, (2012). 
[72] Daniels, J. M, et al. Antibiotics in addition to systemic corticosteroids for acute exacerbations of chronic obstructive pulmonary disease. Am J Respir Crit Care Med, (2010). , 150-157.

[73] Llor, C, et al. Efficacy of Antibiotic Therapy for Acute Exacerbations of Mild to Moderate COPD. Am J Respir Crit Care Med, (2012).

[74] Puhan, M. A, et al. Pulmonary rehabilitation following exacerbations of chronic obstructive pulmonary disease. Cochrane Database Syst Rev, (2011). , CD005305.

[75] Donohue, J. F, et al. A 6-month, placebo-controlled study comparing lung function and health status changes in COPD patients treated with tiotropium or salmeterol. Chest, (2002). , 47-55.

[76] Casaburi, R, et al. A long-term evaluation of once-daily inhaled tiotropium in chronic obstructive pulmonary disease. Eur Respir J, (2002). , 217-224.

[77] Tashkin, D. P, et al. A 4-year trial of tiotropium in chronic obstructive pulmonary disease. N Engl J Med, (2008). , 1543-1554.

[78] Chapman, K. R, et al. Long-term safety and efficacy of indacaterol, a long-acting beta(2)-agonist, in subjects with COPD: a randomized, placebo-controlled study. Chest, (2011). , 68-75.

[79] Stockley, R. A, Chopra, N, \& Rice, L. Addition of salmeterol to existing treatment in patients with COPD: a 12 month study. Thorax, (2006). , 122-128.

[80] Mckeage, K. Indacaterol: a review of its use as maintenance therapy in patients with chronic obstructive pulmonary disease. Drugs, (2012). , 543-563.

[81] Donohue, J. F, et al. Once-daily bronchodilators for chronic obstructive pulmonary disease: indacaterol versus tiotropium. Am J Respir Crit Care Med, (2010). , 155-162.

[82] Dahl, R, et al. Efficacy of a new once-daily long-acting inhaled beta2-agonist indacaterol versus twice-daily formoterol in COPD. Thorax, (2010). , 473-479.

[83] Urzo, D, et al. Efficacy and safety of once-daily NVA237 in patients with moderateto-severe COPD: the GLOW1 trial. Respir Res, (2011). , 156.

[84] Kerwin, E, et al. Efficacy and safety of NVA237 versus placebo and tiotropium in patients with COPD: the GLOW2 study. Eur Respir J, (2012). , 1106-1114.

[85] Van Noord, J. A, et al. QVA149 demonstrates superior bronchodilation compared with indacaterol or placebo in patients with chronic obstructive pulmonary disease. Thorax, (2010). , 1086-1091.

[86] Calverley, P. M, et al. Salmeterol and fluticasone propionate and survival in chronic obstructive pulmonary disease. N Engl J Med, (2007). , 775-789. 
[87] Wedzicha, J. A, et al. The prevention of chronic obstructive pulmonary disease exacerbations by salmeterol/fluticasone propionate or tiotropium bromide. Am J Respir Crit Care Med, (2008). , 19-26.

[88] Jones, P. W, et al. Disease severity and the effect of fluticasone propionate on chronic obstructive pulmonary disease exacerbations. Eur Respir J, (2003). , 68-73.

[89] Zhou, Y, et al. Positive benefits of theophylline in a randomized, double-blind, parallel-group, placebo-controlled study of low-dose, slow-release theophylline in the treatment of COPD for 1 year. Respirology, (2006). , 603-610.

[90] Rossi, A, et al. Comparison of the efficacy, tolerability, and safety of formoterol dry powder and oral, slow-release theophylline in the treatment of COPD. Chest, (2002). , 1058-1069.

[91] ZuWallackR.L., et al., Salmeterol plus theophylline combination therapy in the treatment of COPD. Chest, (2001). , 1661-1670.

[92] Calverley, P. M, et al. Roflumilast in symptomatic chronic obstructive pulmonary disease: two randomised clinical trials. Lancet, (2009). , 685-694.

[93] Fabbri, L. M, et al. Roflumilast in moderate-to-severe chronic obstructive pulmonary disease treated with longacting bronchodilators: two randomised clinical trials. Lancet, (2009). , 695-703.

[94] Retsema, J, et al. Spectrum and mode of action of azithromycin (CP-62,993), a new 15-membered-ring macrolide with improved potency against gram-negative organisms. Antimicrob Agents Chemother, (1987). , 1939-1947.

[95] Williams, J. D, \& Sefton, A. M. Comparison of macrolide antibiotics. J Antimicrob Chemother, (1993). Suppl C: , 11-26.

[96] Bosnar, M, et al. Cellular uptake and efflux of azithromycin, erythromycin, clarithromycin, telithromycin, and cethromycin. Antimicrob Agents Chemother, (2005). , 2372-2377.

[97] Fietta, A, \& Merlini, C. and G. Gialdroni Grassi, Requirements for intracellular accumulation and release of clarithromycin and azithromycin by human phagocytes. J Chemother, (1997). , 23-31.

[98] Foulds, G, Shepard, R. M, \& Johnson, R. B. The pharmacokinetics of azithromycin in human serum and tissues. J Antimicrob Chemother, (1990). Suppl A: , 73-82.

[99] Girard, A. E, et al. Pharmacokinetic and in vivo studies with azithromycin (CP-62,993), a new macrolide with an extended half-life and excellent tissue distribution. Antimicrob Agents Chemother, (1987). , 1948-1954.

[100] Mizukane, R, et al. Comparative in vitro exoenzyme-suppressing activities of azithromycin and other macrolide antibiotics against Pseudomonas aeruginosa. Antimicrob Agents Chemother, (1994). , 528-533. 
[101] Takeoka, K, et al. The in vitro effect of macrolides on the interaction of human polymorphonuclear leukocytes with Pseudomonas aeruginosa in biofilm. Chemotherapy, (1998). , 190-197.

[102] Tateda, K, et al. Potential of macrolide antibiotics to inhibit protein synthesis of Pseudomonas aeruginosa: suppression of virulence factors and stress response. J Infect Chemother, (2000). , 1-7.

[103] Kawamura-sato, K, et al. Postantibiotic suppression effect of macrolides on the expression of flagellin in Pseudomonas aeruginosa and Proteus mirabilis. J Infect Chemother, (2001). , 51-54.

[104] Matsui, H, et al. Azithromycin inhibits the formation of flagellar filaments without suppressing flagellin synthesis in Salmonella enterica serovar typhimurium. Antimicrob Agents Chemother, (2005). , 3396-3403.

[105] Yasuda, H, et al. Interaction between clarithromycin and biofilms formed by Staphylococcus epidermidis. Antimicrob Agents Chemother, (1994). , 138-141.

[106] Starner, T. D, et al. Subinhibitory concentrations of azithromycin decrease nontypeable Haemophilus influenzae biofilm formation and Diminish established biofilms. Antimicrob Agents Chemother, (2008). , 137-145.

[107] Ichimiya, $\mathrm{T}$, et al. The influence of azithromycin on the biofilm formation of Pseudomonas aeruginosa in vitro. Chemotherapy, (1996). , 186-191.

[108] Skindersoe, M. E, et al. Effects of antibiotics on quorum sensing in Pseudomonas aeruginosa. Antimicrob Agents Chemother, (2008). , 3648-3663.

[109] Molinari, G, et al. Inhibition of Pseudomonas aeruginosa virulence factors by subinhibitory concentrations of azithromycin and other macrolide antibiotics. J Antimicrob Chemother, (1993). , 681-688.

[110] Sato, K, et al. Therapeutic effect of erythromycin on influenza virus-induced lung injury in mice. Am J Respir Crit Care Med, (1998). Pt 1): , 853-857.

[111] Suzuki, T, et al. Erythromycin inhibits rhinovirus infection in cultured human tracheal epithelial cells. Am J Respir Crit Care Med, (2002). , 1113-1118.

[112] Yamaya, M, et al. Clarithromycin inhibits type a seasonal influenza virus infection in human airway epithelial cells. J Pharmacol Exp Ther, (2010). , 81-90.

[113] Suzuki, T, et al. Erythromycin and common cold in COPD. Chest, (2001). , 730-733.

[114] Halldorsson, S, et al. Azithromycin maintains airway epithelial integrity during Pseudomonas aeruginosa infection. Am J Respir Cell Mol Biol, (2010). , 62-68.

[115] Asgrimsson, V, et al. Novel effects of azithromycin on tight junction proteins in human airway epithelia. Antimicrob Agents Chemother, (2006). , 1805-1812. 
[116] Hodge, S, et al. Azithromycin improves macrophage phagocytic function and expression of mannose receptor in chronic obstructive pulmonary disease. Am J Respir Crit Care Med, (2008). , 139-148.

[117] Hodge, S, et al. Azithromycin increases phagocytosis of apoptotic bronchial epithelial cells by alveolar macrophages. Eur Respir J, (2006). , 486-495.

[118] Culic, O, et al. Azithromycin modulates neutrophil function and circulating inflammatory mediators in healthy human subjects. Eur J Pharmacol, (2002). , 277-289.

[119] Marjanovic, N, et al. Macrolide antibiotics broadly and distinctively inhibit cytokine and chemokine production by COPD sputum cells in vitro. Pharmacol Res, (2011). , 389-397.

[120] Shimizu, T, \& Shimizu, S. Azithromycin inhibits mucus hypersecretion from airway epithelial cells. Mediators Inflamm, 2012. (2012). , 265714.

[121] Kudoh, S. Applying lessons learned in the treatment of diffuse panbronchiolitis to other chronic inflammatory diseases. Am J Med, (2004). Suppl 9A: , 12S-19S.

[122] Kudoh, S, et al. Improvement of survival in patients with diffuse panbronchiolitis treated with low-dose erythromycin. Am J Respir Crit Care Med, (1998). Pt 1): , 1829-1832.

[123] Fujii, T, et al. Long term effect of erythromycin therapy in patients with chronic Pseudomonas aeruginosa infection. Thorax, (1995). , 1246-1252.

[124] Ichikawa, Y, et al. Reversible airway lesions in diffuse panbronchiolitis. Detection by high-resolution computed tomography. Chest, (1995). , 120-125.

[125] Kadota, J, et al. Long-term efficacy and safety of clarithromycin treatment in patients with diffuse panbronchiolitis. Respir Med, (2003). , 844-850.

[126] Shirai, T, Sato, A, \& Chida, K. Effect of 14-membered ring macrolide therapy on chronic respiratory tract infections and polymorphonuclear leukocyte activity. Intern Med, (1995). , 469-474.

[127] Clement, A, et al. Long term effects of azithromycin in patients with cystic fibrosis: A double blind, placebo controlled trial. Thorax, (2006). , 895-902.

[128] Hansen, C. R, et al. Long-term azitromycin treatment of cystic fibrosis patients with chronic Pseudomonas aeruginosa infection; an observational cohort study. J Cyst Fibros, (2005). , 35-40.

[129] Saiman, L, et al. Azithromycin in patients with cystic fibrosis chronically infected with Pseudomonas aeruginosa: a randomized controlled trial. JAMA, (2003). , 1749-1756.

[130] Pirzada, O. M, et al. Improved lung function and body mass index associated with long-term use of Macrolide antibiotics. J Cyst Fibros, (2003). , 69-71. 
[131] Equi, A, et al. Long term azithromycin in children with cystic fibrosis: a randomised, placebo-controlled crossover trial. Lancet, (2002). , 978-984.

[132] Wolter, J, et al. Effect of long term treatment with azithromycin on disease parameters in cystic fibrosis: a randomised trial. Thorax, (2002). , 212-216.

[133] Southern, K. W, Barker, P. M, \& Solis, A. Macrolide antibiotics for cystic fibrosis. Cochrane Database Syst Rev, (2004). , CD002203.

[134] Wong, C, et al. Azithromycin for prevention of exacerbations in non-cystic fibrosis bronchiectasis (EMBRACE): a randomised, double-blind, placebo-controlled trial. Lancet, (2012). , 660-667.

[135] Altenburg, J, De Graaff, C, Van Der Werf, T, \& Boersma, W. Long term azithromycin treatment: A randomised placebo-controlled trial in non-CF bronchiectasis; results from the BAT trial in European Respiratory Society Congress (2011). Amsterdam.

[136] Banerjee, D, Khair, O. A, \& Honeybourne, D. The effect of oral clarithromycin on health status and sputum bacteriology in stable COPD. Respir Med, (2005). , 208-215.

[137] Seemungal, T. A, et al. Long-term erythromycin therapy is associated with decreased chronic obstructive pulmonary disease exacerbations. Am J Respir Crit Care Med, (2008). , 1139-1147.

[138] Albert, R. K, et al. Azithromycin for Prevention of Exacerbations of COPD. N Engl J Med, (2011). , 689-698.

[139] Shortridge, V. D, et al. Prevalence of macrolide resistance mechanisms in Streptococcus pneumoniae isolates from a multicenter antibiotic resistance surveillance study conducted in the United States in 1994-1995. Clin Infect Dis, (1999). , 1186-1188.

[140] Berg, H. F, et al. Emergence and persistence of macrolide resistance in oropharyngeal flora and elimination of nasal carriage of Staphylococcus aureus after therapy with slow-release clarithromycin: a randomized, double-blind, placebo-controlled study. Antimicrob Agents Chemother, (2004). , 4183-4188.

[141] Malhotra-kumar, S, et al. Effect of azithromycin and clarithromycin therapy on pharyngeal carriage of macrolide-resistant streptococci in healthy volunteers: a randomised, double-blind, placebo-controlled study. Lancet, (2007). , 482-490.

[142] Leclercq, R, \& Courvalin, P. Resistance to macrolides and related antibiotics in Streptococcus pneumoniae. Antimicrob Agents Chemother, (2002). , 2727-2734.

[143] Klugman, K. P, \& Lonks, J. R. Hidden epidemic of macrolide-resistant pneumococci. Emerg Infect Dis, (2005). , 802-807.

[144] Halpern, M. T, et al. Meta-analysis of bacterial resistance to macrolides. J Antimicrob Chemother, (2005). , 748-757. 